\title{
Primal Remonstrance Evolution of NIR Single Chip Optical Sensor for Mental Tomography and their Practicability
}

\author{
Anoop Tiwari \\ Asst.Professor \\ Electronics \& Communication \\ Department, \\ Sam College of Engineering and \\ Technology \\ Rajiv Gandhi Technical University \\ Bhopal (M.P.), India
}

\begin{abstract}
The brain is arguably, the least understood of all the major organs in the human body. While the basic physical structure of the brain and the functioning of separate mental domains and cells inside have been understood for some time, our agreement of the mental functioning as a Research system is still largely taking shape. Here the internal optical signalling based monolithic sensor pops the questions as to feasibility. First, the use of NIR light due to less optical absorption. It shows low signalling level compared to visible light. Another challenge of resolution, due to low signals system designing is also a big call out. Also the small spatial scales of a monolithic IC are a great challenge. In this paper we have discuss the work guarantee the assess the extent of these challenges. A combination of experimental study and computer simulation shows that these challenges can be met and that a monolithic internal optical signalling tomography sensor is fundamentally feasible
\end{abstract}

\section{General Terms}

Optical Sensor Technology

\section{Keywords}

Tomography, internal optical signalling, NIR imaging

\section{INTRODUCTION}

This Brain tomography is most popular technique, and has been helpful for the study of interior picturing of the body. Monitoring the activity of neural assemblage is the key to understanding the functional architecture of brain and coring our knowledge of neurological disease processes.

The techniques which are used for mental tomography are-

1.Optical internal signal (OIS) tomography

2.Functional Magnetic Resonance (FMR)

3.Positron Emission Tomography (PET)

4.Diffuse Optical Imaging (DOI)

5.Spectroscopic techniques

The techniques mentioned above have given the neuroscience community the ability to mental imagery in both human and animal subjects, such as the one shown in figure 1:
These pictures shows similar information to those obtained through multiple electrodes based recording done in past. The classic visual corex maps took few months and years while the new imaging techniques take only few minutes or hours to complete a map due to new techniques are advance especially in speed.

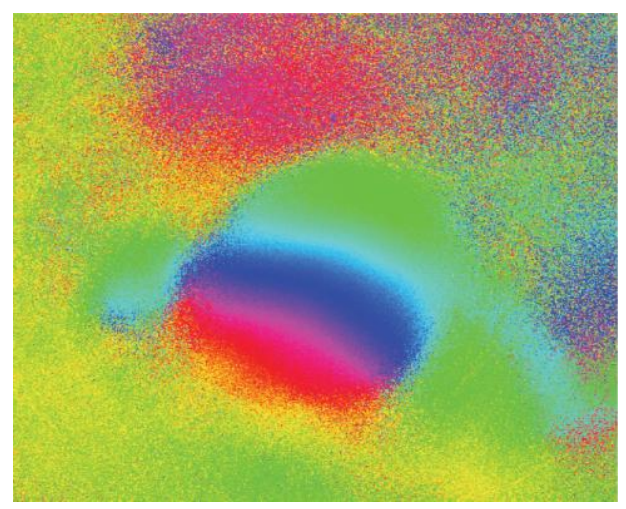

Figure-1: Functional map of mouse primary visual cortex taken using IOS imaging

Here in this research we are using internal optical signalling technique it is also known as optical intrinsic signalling (OIS) or Intrinsic Signal Imaging. This technique is better because of -

1. Inexpensive equipment required

2. Good temporal and spatial resolution

OIS Tomography, is 'intrinsic' cause it doesn't use any other extrinsic marker like fluorescent dyes [11].this technique helps light to trace CBF (cerebrum blood flow), a popular for brain activity. Increased activity in a particular area of the brain causes demand for resources such as 'oxygen 'and 'glucose', which triggers an increase in blood flow to the active area. The increased metabolism and brain tissue, which can be detected as changes in the reflectivity of incident light. Nearinfrared (NIR) imaging techniques are a versatile and increasingly popular means of studying blood flow and oxygenation in human tissue [1]. They exploit the significant 
differences in the absorption spectra of the oxygenated and deoxygenated forms of haemoglobin at near-infrared wavelengths. By measuring the changes in the intensity of diffusely transmitted near-infrared light across a region of tissue it is Possible to gain well-localized information about blood oxygenation and hemodynamic. Optical configuration is the application of NIR techniques to produce spatially resolved, Two-dimensional images of changes in both oxy haemoglobin (HbO2) and de-oxy haemoglobin ( $\mathrm{HbR})$.

The monolithic IC NIR IOS sensor poses some fundamental questions as to Feasibility. First, the use of NIR light, where optical absorption is low, means that the IOS is almost entirely dependent on scattering changes to provide contrast between active and inactive brain regions. This result in signal levels that are low compared to IOS imaging using visible light. It also raises questions of resolution, as the increased role of scattering can cause blurring of images. The low signals also present challenges for system and component design. The Fundamental system requirements must be evaluated and understood. Light sources must be sufficiently stable and detectors must have sufficiently low dark current so as to not overwhelm the IOS. These requirements will have an impact on choices of Semiconductor material, source and detector type, and array architecture. Finally, the small spatial scales of a semiconductor chip are such that they fall within 1-2 photons mean free paths. This raises a concern that photons will not undergo sufficient scattering events in order to be detected, or that the detected photons will not have penetrated to sufficient depth within the tissue to give meaningful IOS images. This work undertaken the asses the magnitude of these challenges. A combination of empirical study and computer simulation shows that these challenges can be met, and that a single-chip IOS imaging sensor is fundamentally feasible.

Figure-2: Optical Tomography vs. standard imaging

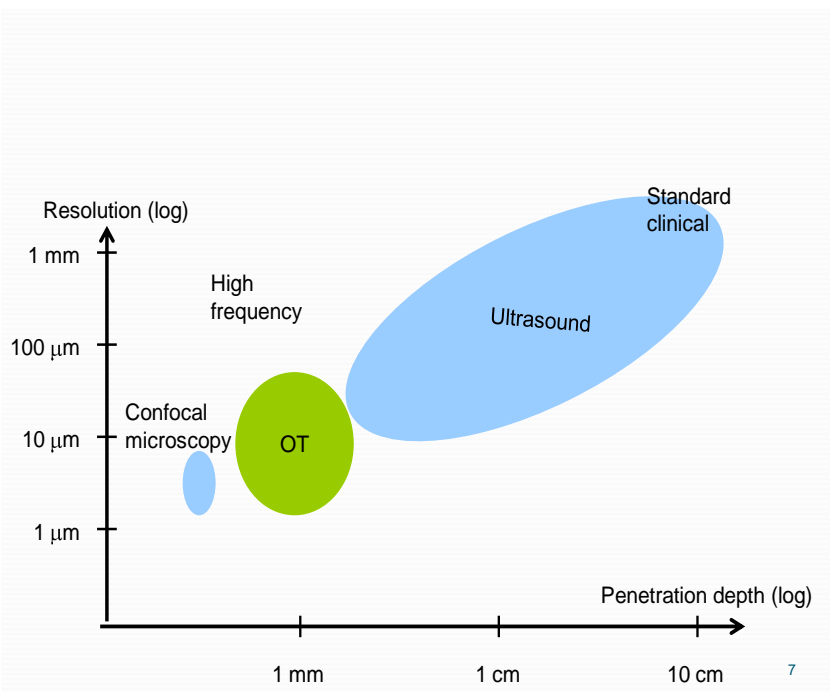

\section{TOMOGRAPHY METHODOLOGY OF NIR IOS}

To develop the proposed system, it is necessary to study the characteristics of IOS at far-red and near-infrared wavelengths. In particular, we are interested in the relative intensity change of the backscattered light $(\Delta R / R)$ from a given region of the brain between periods of activity and inactivity. The relative intensity change is influenced by the absorption of light by blood, which is dominated by oxyhaemoglobin, deoxyhemoglobin and water. Visible wavelengths typically used for IOS imaging $(510 \mathrm{~nm}$ to $650 \mathrm{~nm})$ are absorbed more strongly than NIR light $(690 \mathrm{~nm}$ to $850 \mathrm{~nm})$, and thus produce stronger intensity changes. $[4,9,10]$ However, higher absorption also limits penetration depth, requiring visible light IOS images to be taken through a craniotomy. NIR light encounters significantly less absorption than visible light, allowing for the possibility of IOS imaging through the skull. The last several years has seen considerable interest in the use of NIR light for minimally invasive imaging of human and animal subjects using fibre-based systems. [5,7] One disadvantage of reduced absorption is that photons will experience more scattering events before being detected. This raises the background intensity and delocalizes the signals, making them more difficult to detect and maps more difficult to resolve. This work seeks to understand the implications of imaging IOS at near infrared wavelengths by comparing maps taken at selected wavelengths between $610 \mathrm{~nm}$ (red) and $850 \mathrm{~nm}$ (NIR). Additionally, we seek to observe and understand the effects of scattering through the skull by comparing maps taken with and without the skull removed.

The experimental setup is shown in Figure 3.
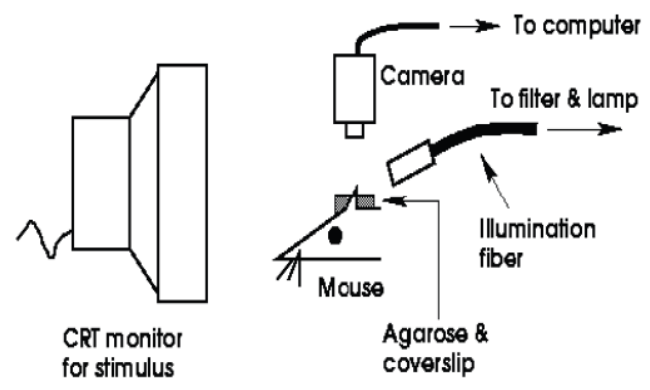

Fig.-3: Imaging setup [1]

An anesthetized C57BL6 wild-type mouse is given a visual stimulus from a computer monitor consisting of a horizontal white stripe on a grey background (50\% contrast.) images are obtained by illuminating the visual cortex at $610 \mathrm{~nm}, 690 \mathrm{~nm}$, $750 \mathrm{~nm}, 775 \mathrm{~nm}$, or $850 \mathrm{~nm}$. Light from a tungsten lamp is filtered at a given wavelength using interference filters with a FWHM bandwidth of $10 \mathrm{~nm}$ and delivered via an optical fibre. Images without a craniotomy (but with scalp removed) are taken first, then a small section of skull above the visual cortex is removed and images taken again. The stimulus is swept 
repeatedly in elevation across the visual field with a period of 8 sec/sweep for 90 cycles (Figure 4), with a DALSA 1M30 CCD camera (DALSA Corporation, Waterloo, Ontario, Canada) capturing images at 30 frames per second.

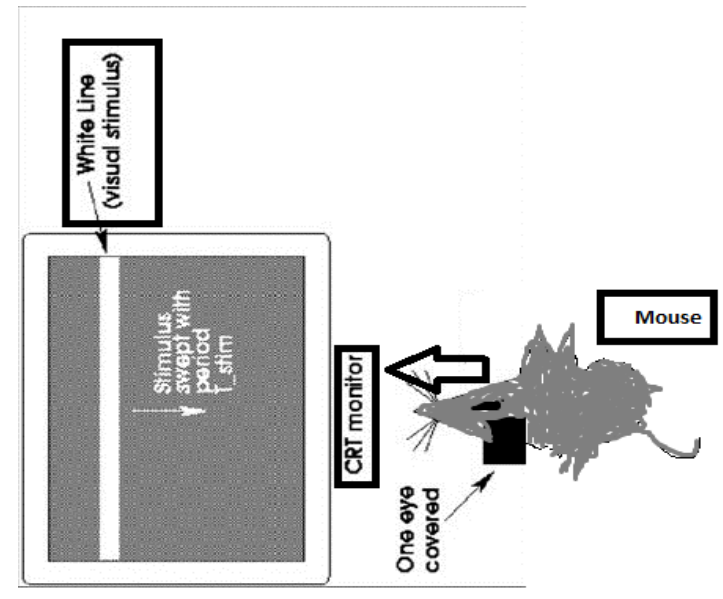

Figure-4: Visual stimulus[12]

The stimulus is then swept in the opposite direction and images taken. Signals recorded from the two sweeps are then subtracted to remove shifts caused by hemodynamic delay. After the images are recorded, a Fourier transform in time is performed for each pixel, and the signal is filtered for components at the sweep frequency and normalized to improve the signal to noise ratio (Figure 5), the result is two maps, one of signal.

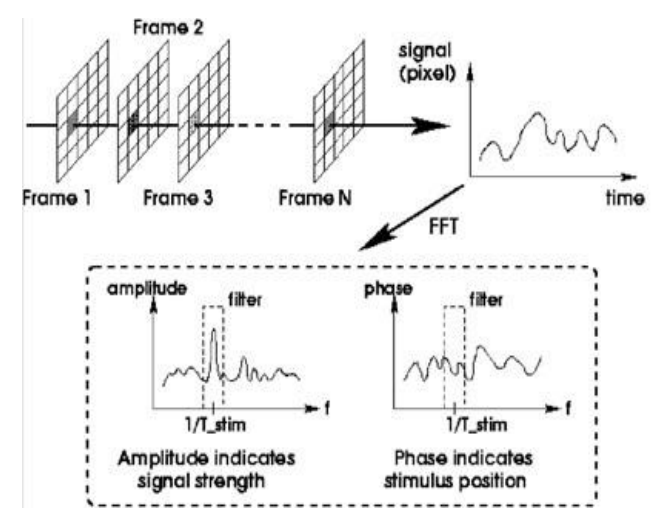

Figure-5: Image processing: A pixel-by-pixel FFT is used to select only the component at the stimulus frequency, thereby filtering out noise from other physiological and physical processes Amplitude, indicating the relative intensity change, and one of phase, corresponding to the position of the stimulus within the visual field. Animals are euthanized after the entire set of pictures is taken.

\section{RESULTS OF TOMOGRAPHY}

The images obtained without outlier elimination registered on the brain is shown in fig.6 and with outlier elimination registered on the brain is shown in fig. 7 respectively..
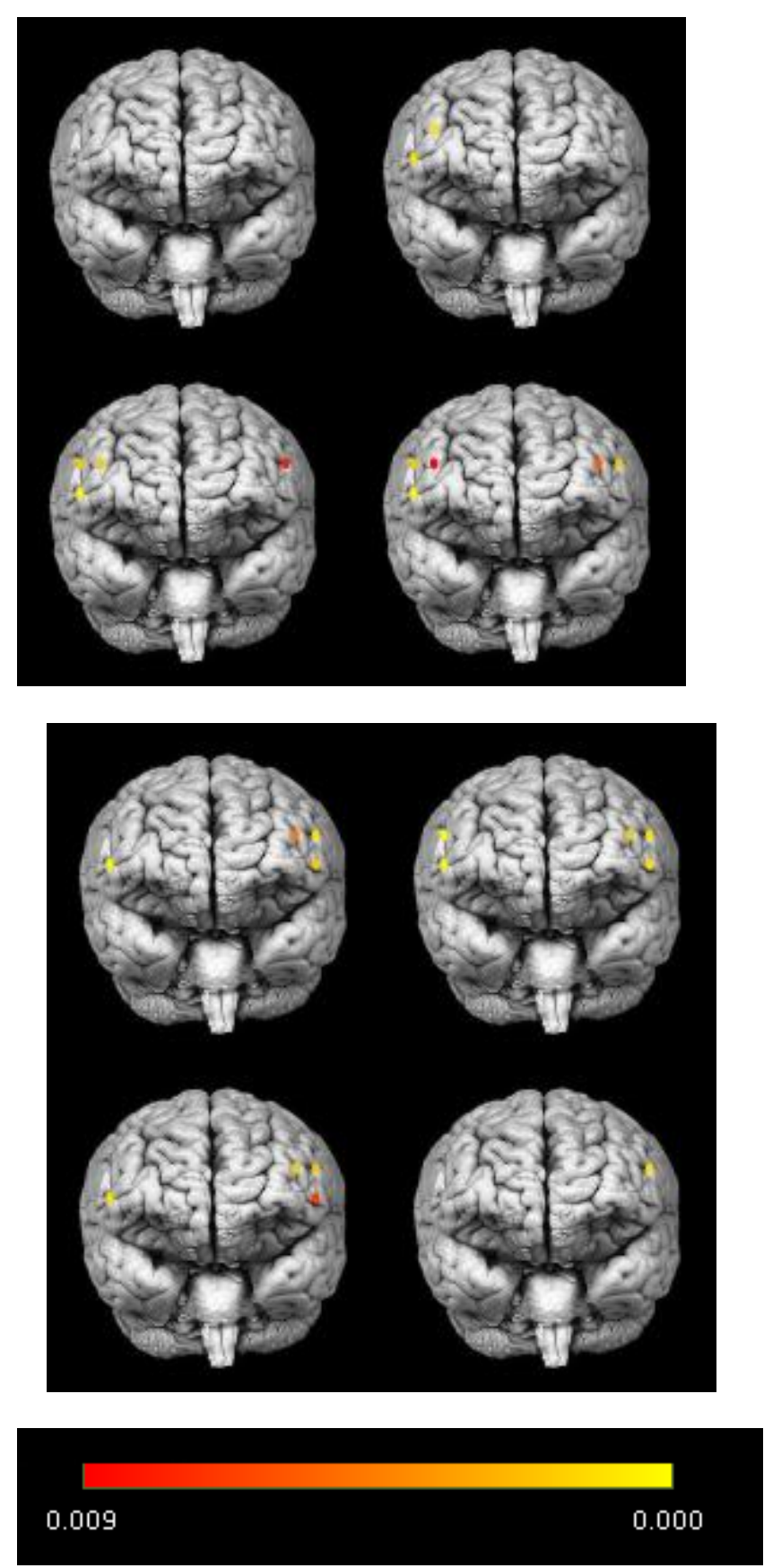

Figure 6: Analysis results without outlier elimination registered on the brain 

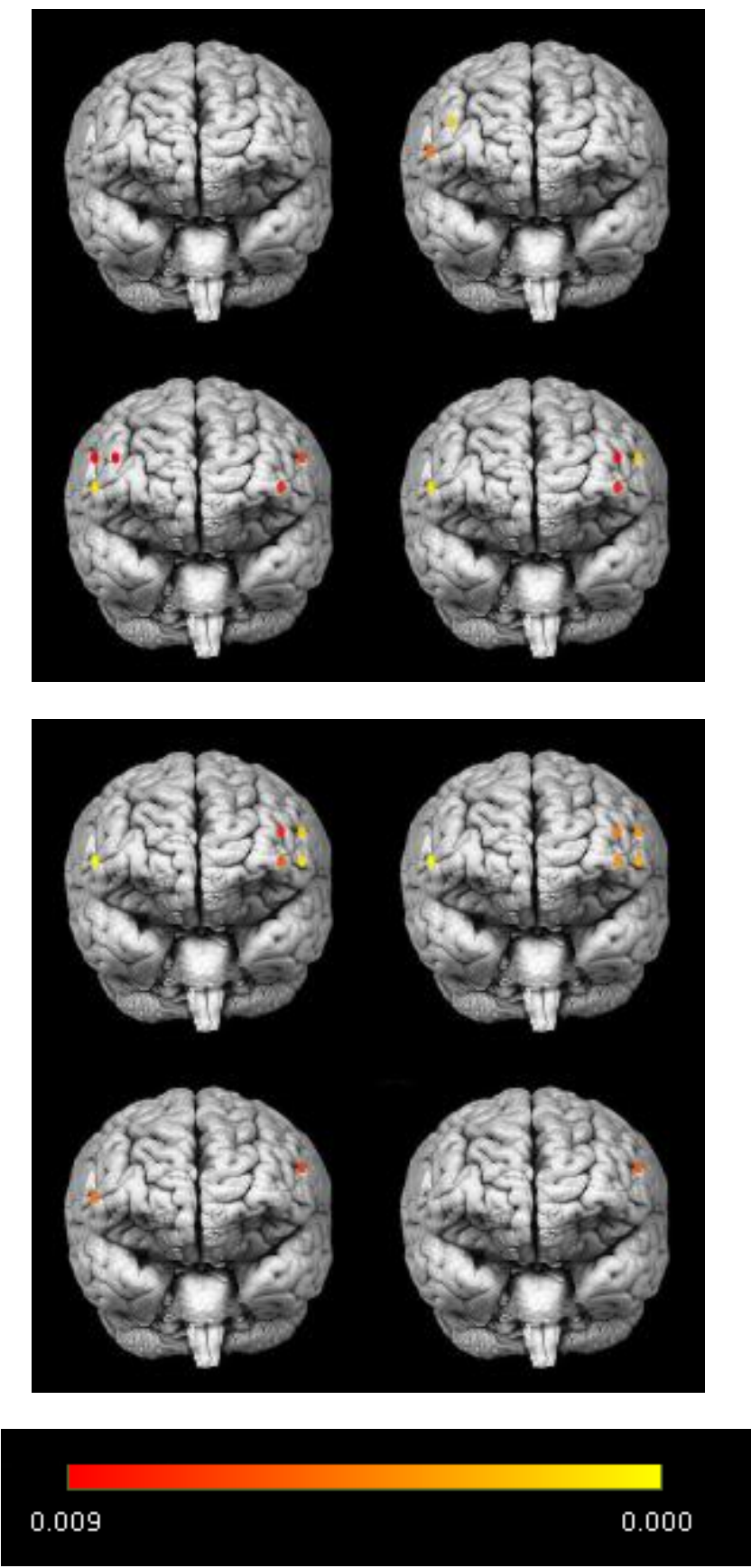

Figure 7: Analysis results with outlier elimination registered on the brain

In above figures, each set of images from left to right was taken at increasing level of wavelengths. It is observable from the fading black region in the amplitude images that the signal to background ratio decreases as wavelength moves from visible to NIR. Figure- 8 shows that are because of degradation in detected signal level rather than an increase in background. In the figure given just below shows that, In NIR signal to background ratio is approximately 2-3.Imaging through skull causes a signal loss of $15 \%-20 \%$. The change in reflectivity is
$\Delta \mathrm{R} / \mathrm{R}=5 \times 10^{-5}$ or $-86 \mathrm{~dB}$.

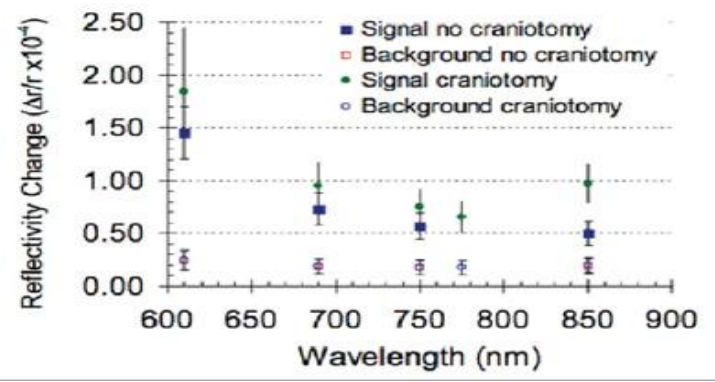

Figure-8: Signal to background analysis. Decreasing signal to background ratio is because of decreasing signal rather than increasing background.

\section{TOMOGRAPHY OVER SHORT DISTANCES}

The short distances separating sources and detectors presents an additional fundamental question regarding the feasibility of monolithic IOS tomography. Due to IOS imaging depends on backscattered light, the penetration depth of detected light is proportional to distance between the source and detector. Since chip-scale dimensions are quite small, this raises a concern that a sensor placed outside the skull will not penetrate deep enough to reach the brain.

\section{VCSEL NOISE TESTING}

Meeting this dynamic range requirement presents the need for a powerful, low-noise light source. Vertical Cavity Surface Emitting Lasers (VCSELs) designed for single mode Operation over a middle ground between LEDs and multi-mode VCSELs [3] and are easily incorporated into an integrated emitter/photodiode system [2,8]. The figure of merit used to characterize intensity noise is known as Relative Intensity Noise (RIN). It is expressed as the ratio of the mean-square noise power in a $1 \mathrm{~Hz}$ bandwidth to the square of the average optical power. The average photocurrent in the detector. [6]

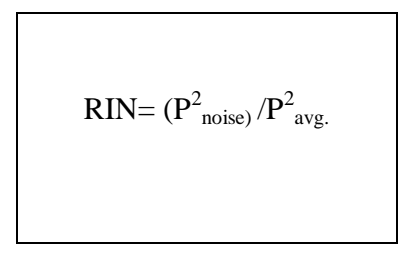

The low-frequency RIN performance of VCSELs is more than sufficient for the purposes of IOS tomography. The peak RIN for the commercial VCSEL measured in this work is $-85 \mathrm{~dB}$ at the device threshold current, but can be reduced to as low as $153 \mathrm{~dB}$ by increasing the drive current of the VCSEL. At $10 \mathrm{~Hz}$, RIN ranges from -196 to $-174 \mathrm{~dB} / \mathrm{Hz}$. The source of peaks in the RIN spectrum from $10-200 \mathrm{~Hz}$ has been traced to interference from other high-power building facilities equipment. 


\section{CONCLUSION}

We have seen that a monolithic tomography system is possible from fundamental points of view. NIR Tomography through the skull of a mouse has been shown to produce operational images and light in a system with short source/detector separation is shown to have sufficient penetration depth to detect blood flow in the cortex of a mouse. at long last, it has been shown that VCSEL can be made sufficiently stable to be used as the light source for internal optical signalling tomography.

\section{REFERENCES}

[1]. Thomas T. Lee, Ofer Levi, Jianhua Cang, Megumi Kaneko, Michael P. Stryker, Stephen J Smith, Krishna V. Shenoy, and James S. Harris, Integrated Semiconductor Optical Sensors for Chronic, Minimally-Invasive Imaging of Brain Function EMBS Annual International Conference New York City, USA, Aug 30-Sept 3, 2006

[2]. A. M. Kasten, A. V. Giannopoulos, C. Long, C. Chen, and K. Choquette, "Fabrication and characterization of individually addressable vertical-cavity surface emitting laser arrays and integrated VCSEL/PIN detector arrays", Proceedings of the SPIE, vol. 6484, pp. 6484C -1 -6484C6, 2007.

[3] O. Levi, T. T. Lee, M. M. Lee, S. J. Smith, and J. S. Harris, "Integrated semiconductor optical sensors for cellular and neural imaging," Applied Optics, vol. 46, no. 10, pp. 18811889, 2006

[4] A. Dunn, A. Devor, A. Dale, and D. Boas, ISpatial extent of oxygenation metabolism and hemodynamic changes during functional activation of rat somatosensory cortex'," NeuroImage, vol. 27, no. 2, pp. 279\{290, 2005.

[5] K. Tanner, E. D'Amico, A. Kaczmarowski, S. Kukreti, J. Malpeli, W. Mantulin, and E. Gratton, ISpectrally resoloved neurophotonics: a case report of hemodynamics and vascular components in the mammalian brain," Journal of Biomedical Optics, vol. 10, no. 6, p. 064009, 2005.

[6] L. Goddard, ICharacterization and modeling of the intrinsic properties of $1.5 \mathrm{~m}$ GaInNAsSb/GaAs lasers," Ph.D. Dissertation, Stanford University, 2005.

[7] M. Francheschini and D. Boas, INoninvasive measurement of neuronal activity with near-infrared optical imaging," NeuroImage, vol. 21, no. 1, pp. 372\{386, 2004.

[8 ] E. Thrush, O. Levi, W. Ha, G. Carey, L. J. Cook, J. Deich, S. J. Smith,W. Moerner, and J. S. Harris, Integrated semiconductor vertical-cavity surface emitting lasers and PIN photo detectors for biomedical putrescence sensing, "IEEE Journal of Quantum Electronics, vol. 40, no. 5, pp. $491\{497,2004$.

[9] E. Shtoyerman, A. Arieli, H. Slovin, I. Vanzetta, and A. Grinvald, "Long-term optical imaging and spectroscopy reveal mechanisms underlying the intrinsicsignal and stability of cortical maps in v1 of behaving monkeys", Journal of Neuroscience, vol. 20, no. 21, pp. 8111-8121, 2000

[10] S. Prahl. (1998) Tabulated molar extinction coe_cient for hemoglobin in water. [Online]. Available: http://omlc.ogi.edu/spectra/hemoglobin/summary.html

[11] D. H. Hubel and T. N. Wiesel, "Receptive fields, binocular interaction, and functional architecture in the cat's visual cortex,"Journal of Physiology (Lon-don), vol. 160, pp. 106154, 1962.

[12] Anoop Tiwari, Ravi Shankar mishra ,Rajesh Nema "Design of optical internal signaling based optical sensor for encroaching mental tomography" Internation journal of engineering, science and technology,vol.3, march 2011,p.p. 2454-2460. 